\title{
DAIR: A Query-Efficient Decision-based Attack on Image Retrieval Systems
}

\author{
Mingyang Chen* \\ University of New South Wales \\ Sydney, Australia \\ mingyang.chen1@unsw.edu.au
}

\author{
Junda $\mathrm{Lu}^{*}$ \\ University of New South Wales \\ Sydney, Australia \\ junda.lu@student.unsw.edu.au
}

\author{
Yi Wang ${ }^{\dagger}$ \\ Dongguan University of Technology \\ Dongguan, China \\ wangyi@dgut.edu.cn
}

\author{
Jianbin Qin ${ }^{\ddagger}$ \\ Shenzhen Institute of Computing \\ Sciences, Shenzhen University \\ Shenzhen, China \\ qinjianbin@szu.edu.cn
}

\author{
Wei Wang§ \\ University of New South Wales \\ Sydney, Australia \\ weiw@cse.unsw.edu.au
}

\begin{abstract}
There is an increasing interest in studying adversarial attacks on image retrieval systems. However, most of the existing attack methods are based on the white-box setting, where the attackers have access to all the model and database details, which is a strong assumption for practical attacks. The generic transfer-based attack also requires substantial resources yet the effect was shown to be unreliable.

In this paper, we make the first attempt in proposing a queryefficient decision-based attack framework for the image retrieval (DAIR) to completely subvert the top- $K$ retrieval results with humanimperceptible perturbations. We propose an optimization-based method with a smoothed utility function to overcome the challenging discrete nature of the problem. To further improve the query efficiency, we propose a novel sampling method that can achieve the transferability between the surrogate and the target model efficiently. Our comprehensive experimental evaluation on the benchmark datasets shows that our DAIR method outperforms significantly the state-of-the-art decision-based methods. We also demonstrate that real image retrieval engines (Bing Visual Search and Face++ engines) can be attacked successfully with only several hundreds of queries.
\end{abstract}

\section{CCS CONCEPTS}

- Computing methodologies $\rightarrow$ Computer vision problems; - Security and privacy $\rightarrow$ Software and application security.

\footnotetext{
${ }^{*}$ The first two authors have equal contributions.

†ORCID of Yi Wang: 0000-0002-8448-8570.

¥ORCID of Jianbin Qin: 0000-0002-4298-3215

$\S$ Corresponding author.
}

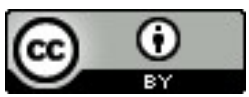

This work is licensed under a Creative Commons Attribution International 4.0 License. SIGIR '21, July 11-15, 2021, Virtual Event, Canada. (C) 2021 Copyright held by the owner/author(s). ACM ISBN 978-1-4503-8037-9/21/07.

https://doi.org/10.1145/3404835.3462887

\section{KEYWORDS}

Content-based Image Retrieval, Adversarial Attack in Deep Learning, Decision-based Attack in Deep Learning

ACM Reference Format:

Mingyang Chen, Junda Lu, Yi Wang, Jianbin Qin, and Wei Wang. 2021. DAIR: A Query-Efficient Decision-based Attack on Image Retrieval Systems. In Proceedings of the 44th International ACM SIGIR Conference on Research and Development in Information Retrieval (SIGIR '21), July 11-15, 2021, Virtual Event, Canada. ACM, New York, NY, USA, 10 pages. https://doi.org/10.1145/ 3404835.3462887

\section{INTRODUCTION}

Deep Neural Network (DNN) has been widely employed in various fields and has become the core technologies of many industries such as autonomous driving [6, 28], machine translation [15, 41], and image recognition [16, 37]. However, Szegedy et al. [38] found that even state-of-the-art image classifiers can still be misled by a malicious human imperceptible perturbation. To investigate the intrinsic defects in DNN-based models, an increasing number of research works are proposed to design imperceptible perturbations injecting on the raw input to fool the decisions of attacked models. This work is known as the adversarial attack.

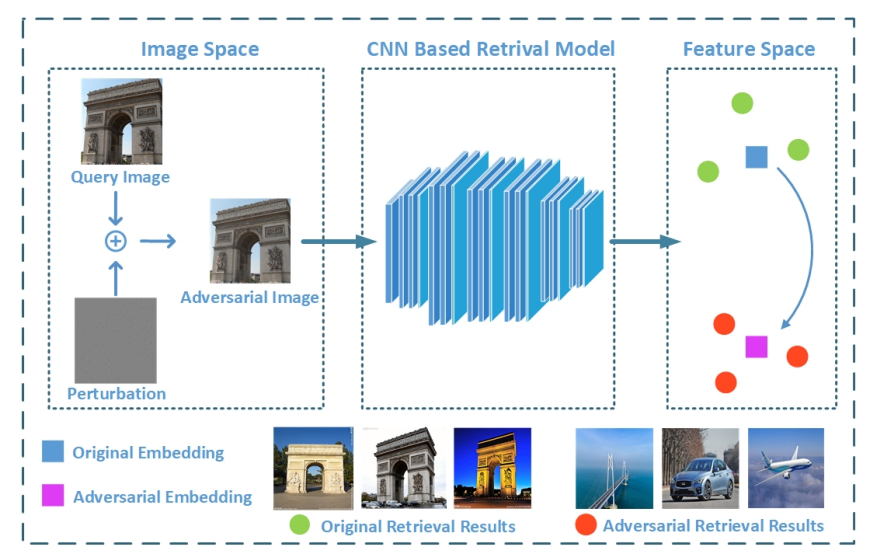

Figure 1: Overview of attacking image retrieval systems. 
While there has been a wide body of literature on adversarial attacks on classification models, there is increasing interest in studying adversarial attacks to the image retrieval system, especially those employing DNNs as feature extractors [23, 26, 39, 47, 50]. Fig. 1 illustrates the overall process, where the attacker can compute an adversarial perturbation and superimpose it on the original query image, such that the top- $K$ returned images of the adversarial queries are totally different from those of the original query.

We categorize existing adversarial attacks on image retrieval models into two categories based on their requirement of accessible resources: the white-box attack and the black-box attack. For whitebox attacks, attackers have access to all the model details, including the model architecture and parameters, and all or a large subset of images in the databases. Methods in this category employ objective functions based on the embeddings of the query and database images, and use backpropagation to compute the adversarial query images [26, 39, 47, 50]. For the black-box attack, existing methods are based on the transferred attack. They do not require model or database details, but do require access to a set of images with the same or highly similar distribution as the database images. For example, [23] trains an input-agnostic adversarial perturbation pattern based on surrogate models and demonstrated its transferability. There are several limitations to the above existing approaches. Methods in the white-box attack category have a stringent requirement and unlikely to be practical. The transfer-based attacks may be more realistic, but they still require a substantial amount of high-quality training data or knowing the detailed structure of the target model, and their success rates are unpredictable ${ }^{1}$.

In this paper, we study the problem of attacking image retrieval systems in a black-box setting, with the mild assumption that the attacker can issue multiple image retrieval queries to the system and obtain the identities of the top- $K$ returned images ${ }^{2}$. This setting is most similar to the decision-based attack methods for classification models where only the class labels are required to launch a successful attack. We first demonstrate that existing state-of-the-art decision-based attack methods do not work in our problem setting, due to the discrete nature of the problem. To tackle this challenge, we adapt the Projected Natural Evolution Strategy to our problem by proposing the new projection operator and utility functions, as well as putting forward several optimization techniques that help to reduce the magnitude of the adversarial perturbations needed. Moreover, we propose novel subspace sampling strategies based on surrogate models and dropout techniques to further improve the effectiveness of the proposed method. We evaluate the performance of our proposed techniques with several baseline methods on the standard image retrieval benchmark datasets. We also demonstrate the practicality of our proposed technique by attacking two commercial image retrieval systems across a variety of settings.

\section{RELATED WORK}

\subsection{Deep-Feature-based Image Retrieval}

Content-Based Image Retrieval (CBIR) aims to find the most related or similar images for a specific query from a large set of candidates. With the developments in Convolutional Neural Network (CNN)

\footnotetext{
${ }^{1}$ For example, see Table 3.

${ }^{2}$ Note that we do not require the top- $K$ images themselves.
}

models [19, 27], recent works of CBIR [42, 46] proposed to use CNN models as feature extractors and gained superior improvements. To further boost the feature representation and global semantic preserving, many recent works proposed to study the trainable pooling layers, which can refine the feature embedding output by the CNN models $[1,18,33,40]$. In this paper, we aggregated VGGNet [36] and ResNet [16] with two state-of-the-art pooling methods MAC [40] and GeM [33] as our deep retrieval models to evaluate the attacking performances. These two models are widely used in the benchmark tests of current existing image retrieval attacks $[23,48]$.

\subsection{Adversarial Attacks in Classification}

The adversarial attacks for classification models can be divided into two categories which are white-box and black-box attacks. White-box attacks, which are also known as optimization-based attacks, allow attackers to access all the resources of the target model. Subsequently, attackers regard the attack as an optimization problem and design various objective functions to derive adversarial perturbations by the backpropagation. Examples include L-BFGS [38], the Fast Gradient Sign Method (FGSM [14]), the Basic Iterative Method (BIM [21]), Deepfool [31], and the CW attack [5].

The black-box attacks can be further divided into three types which are transfer-based, score-based, and decision-based attacks. Transfer-based attacks utilize the transferability between different models to generate an adversarial perturbation on the surrogate model to degrade the prediction of the target model [25, 30]. Scorebased attacks $[8,24]$ assume that the attackers can observe the logit scores output by the target classification model. Therefore, score-based attacks are generally optimization-based but are solved by zero-order optimization methods, such as the finite difference method or the Natural Evolutionary Strategies (NES [17]). The decision-based attacks require only the hard-label output by the target models, which is more realistic in practice. Thus our work focus on this attack setting. [2] firstly prosed the Boundary Attack (BA) to find the minimum perturbation by a series of random walks on the decision boundary. OPT-Attack [9] and Sign-OPT-Attack [10] turn the attack goal of finding the minimum adversarial perturbation to find the direction originated from the target image with the minimum boundary distance. The HopSkipJump attack (HSJA [7]) approximates the gradient of logit scores on the decision boundary. Besides, [13] proposed the Evolutionary Attack (EA) with covariance matrix adaptation for face recognition models employing CNN-based retrieval models to extract the deep feature embeddings of face images. Nevertheless, it should be noticed that EA regards the attacked face recognition models as binary classifiers to output the matching result of a query face image, which is different from our setting.

\subsection{Adversarial Attacks in Retrieval}

Unlike the classification models which can independently make the prediction based only on the output logit scores of the target query, the ranking list predicted by the retrieval model is related to all the candidate images. Consequently, multiple gradient-based attacks for retrieval models proposed to find the directions which can quickly push the feature embedding of the target query away 
from its own area or drag it to be close to some target areas. Among them, [50] proposed the Candidate Attack (CA) and the Query Attack (QA) to manipulate the ranking result by increasing or decreasing the distance of the adversarial embedding to certain target embeddings. [39] proposed to implement the targeted mismatch adversarial attack by decreasing the distance of a carrier image to the target image in the image space as well as in the embedding space. [47] proposed to degrade the retrieval quality of a query by maximizing the Hamming distance to its own embedding. In terms of black-box attacks, some transfer-based attacks are proposed. Inspired by the universal adversarial perturbations (UAPs [30]), [23] proposed to train an input-agnostic adversarial perturbation on surrogate models with unrelated datasets, which gained significant degradation of ranking metrics of the target model. However, there are two shortcomings of this transfer-based attack. Firstly, the crafted perturbations commonly have longish lengths and structured noise patterns, which are more perceptible to humans after injecting on the queries. Secondly, it is unreliable to attack with a specific goal, i.e. subverting all the top- $K$ retrieval results.

\section{PROPOSED DAIR METHOD}

In this section, we formally define the decision-based attack for image retrieval models (DAIR), followed by its technical challenge. We then outline our attack method based on a Projected NES algorithm framework, followed by various optimizations.

\subsection{Problem Definition and Preliminaries}

We model an image retrieval system by a function $F(\cdot)$ that takes in a query image $\mathbf{x}^{*} \in[0,1]^{d}$, and returns top- $K$ most similar images from a backend image database $\mathcal{D} \mathcal{B}$. The goal of DAIR is to find an adversarial image $\mathbf{x}^{\text {adv }}$ that is indistinguishable from $\mathbf{x}^{*}$, yet its retrieval results are totally different from that of $x^{*}$. This can be formally formulated as the following constrained optimization problem:

$$
\begin{array}{ll}
\text { minimize } & D\left(\mathbf{x}^{\mathrm{adv}}, \mathbf{x}^{*}\right) \\
\text { subject to } & F\left(\mathbf{x}^{\mathrm{adv}}\right) \cap F\left(\mathbf{x}^{*}\right)=\emptyset
\end{array}
$$

where $D(\cdot, \cdot)$ is a function that measures the dissimilarity between two input images. Hereafter, we will use the Euclidean distance as the $D(\cdot, \cdot)$ function as it is one of the most prevalent choices in previous works $[22,26]$. The constraint we consider here is stringent that requires the top- $K$ images of the original query are all subverted by the adversarial image. We note that our problem definition and our proposed solutions can be easily extended to consider other dissimilarity functions or other constraints, such as the intersection size is upper bounded by $\epsilon \cdot K$, for any constant $\epsilon \in[0,1)$.

We abbreviate the constraint in Equation (1) as $C\left(\mathrm{x}^{\mathrm{adv}}\right)$ and it evaluates to either True or False. It induces the infeasible region, which is a closed set $\Omega_{-} \stackrel{\text { def }}{=}\left\{1 \llbracket \neg C(\mathbf{x}) \rrbracket, \mathbf{x} \in[0,1]^{d}\right\}$, where $1 \llbracket p \rrbracket$ is the indicator function using the predicate $p$. It also induces the feasible region, which is an open set $\Omega_{+} \stackrel{\text { def }}{=}\left\{\mathbf{1} \llbracket C(\mathbf{x}) \rrbracket, \mathbf{x} \in[0,1]^{d}\right\}$. We will refer to the boundary of $\Omega_{+}$simply as the boundary.

We assume the attacker has no access to the model detail or any image set that resembles the images in the backend database. We only assume that the attack algorithm has the unique identifier of the top- $K$ images returned by the retrieval model, but not the top- $K$ images themselves ${ }^{3}$. While this is a significantly more challenging setting than existing white-box attacks to image retrieval models $[26,47,50]$, such setting is practical and generally allows us to apply our methods to real-world image retrieval systems.

\subsection{Technical Challenges}

HSJA [7], or its variants (such as QEBA [22]), is a state-of-the-art decision-based black-box attack algorithm for classification models requiring significantly fewer queries than the previous decisionbased attack methods such as the Boundary Attack [2] or OptAttack [9].

We note that HSJA cannot be directly applied in our problem settings. This is mainly because the convergence of the algorithm requires the existence of a score function $S$ with the property that its gradient is bounded away from 0 near the boundary from within $\Omega_{-}$. For classification models, the function $S(\mathbf{x})$ is trivially a logit score based function w.r.t the input $\mathbf{x}$, and it generally satisfies this technical condition.

To characterize this inapplicability, let us assume that, without loss of generality, the image retrieval system computes a score for each database image $\mathbf{o}_{i}$ with respect to a query image $\mathbf{x}$ via a function $f\left(\mathbf{x}, \mathbf{o}_{i}\right)$. It then returns the top- $K$ images with the highest scores. We also assume $f(\cdot ; \mathbf{o})$ is Liptschitz $\forall \mathbf{o}$, i.e., there exist a constant $L$, such that $|f(\mathbf{x} ; \mathbf{o})-f(\mathbf{y} ; \mathbf{o})| \leq L \cdot\|\mathbf{x}-\mathbf{y}\|$. Then the following Lemma shows that any non-trivial function based on the retrieval results has a gradient of 0 at many places within $\Omega_{-}$, including the vicinity of the boundary.

LEMMA 1. For any non-trivial score function defined on the retrieval results, i.e., $S(\mathbf{x})=f(F(\mathbf{x}))$, there exists infinite number of points $\mathbf{x}$ near the boundary such that $\nabla_{\mathbf{x}}(\mathbf{x})=\mathbf{0}$.

\subsection{Projected Natural Evolution Framework}

The intuitive reason for the inability to apply the HSJA algorithm for DAIR is because of the discrete nature of the top- $K$ retrieval results and this induces a step-like function in $\Omega_{-}$. To overcome this challenge, we adapt the Projected Natural Evolution Strategy (PNES) method [17] to compute a direction to move the current $\mathbf{x}^{\text {adv }}$ to a location in $\Omega_{+}$. PNES has demonstrated better performance than alternative methods [13].

On a high level, the PNES method defines a smoothed function over $\Omega_{-}$such that its gradient exists. This allows it to use the projected gradient descent (PGD) method [29] to solve the constrained optimization problem (Eq. (1)). More specifically, let $\mu(\mathbf{x})$ be a utility function of an image $\mathbf{x}$ based on $F(\mathbf{x})$. We define its smoothed corresponding function:

$$
J(\mathbf{x})=\mathbf{E}_{\boldsymbol{\epsilon} \sim \mathrm{N}(0, \mathbf{I})} \llbracket \mu(\mathbf{x}+\sigma \cdot \boldsymbol{\epsilon}) \rrbracket
$$

where $\sigma$ is a hyper-parameter that controls the magnitude of the random Gaussian disturbance $\sigma \cdot \boldsymbol{\epsilon}$. The Natural Evolution Strategy (NES) algorithm [43] computes the gradient of $J(\mathbf{x})$ based on the

\footnotetext{
${ }^{3}$ This assumption is satisified by many real-world image retrieval system. E.g., we can use the returned urls of the images as the unique identifiers as in Google's image search.
} 
log-likelihood trick:

$$
\begin{aligned}
\boldsymbol{\nabla}_{\mathbf{x}} J(\mathbf{x}) & =\frac{1}{\sigma} \mathrm{E}_{\boldsymbol{\epsilon} \sim \mathrm{N}(0, \mathbf{I})} \llbracket \mu(\mathbf{x}+\sigma \cdot \boldsymbol{\epsilon}) \cdot \boldsymbol{\epsilon} \rrbracket \\
& \approx \frac{1}{\sigma \cdot B} \sum_{i=1}^{B} \mu\left(\mathbf{x}+\sigma \cdot \boldsymbol{\epsilon}_{i}\right) \cdot \boldsymbol{\epsilon}_{i}
\end{aligned}
$$

Eq. (4) uses Monte Carlo method to obtain an estimate of Eq. (3) using $B$ random samples $\boldsymbol{\epsilon}_{i}$.

The final PNES algorithm works in an iterative fashion: (i) it starts with an initial $\mathbf{x}^{(0)}$ that lies on the boundary, and (ii) in each iteration, it obtains the next $\mathbf{x}^{(t+1)}$ by first moving along the direction of the gradient (Equation (4)) and then projecting it back onto the feasible region:

$$
\mathbf{x}^{(t+1)}=\prod\left(\mathbf{x}^{(t)}-\eta \cdot \nabla_{\mathbf{x}^{(t)}} J\left(\mathbf{x}^{(t)}\right)\right)
$$

where $\boldsymbol{\nabla}_{\mathbf{x}^{(t)}} J\left(\mathbf{x}^{(t)}\right)$ is estimated using NES, and $\eta$ is a hyper-parameter specifying the step size. The role of the projection operator, $\Pi(\cdot)$, is to maintain the invariant that the current $\mathbf{x}^{(t)}$ always satisfies the constraint.

\subsection{Adapting PNES to DAIR}

3.4.1 The Projection Operator. The original PNES algorithm [17] only targets at the simple constraint that $\mathbf{x}^{(t+1)}$ must reside within an $\epsilon$ ball (measured in $\ell_{\infty}$ norm) around $\mathrm{x}^{*}$ and hence the projection operator is simple a clipping function.

Considering the objective function of our new optimization problem, we define the projection operator, denoted as $\Pi_{C, \mathbf{p}}(\mathbf{x})$, as:

$$
\Pi_{C, \mathbf{p}}(\mathbf{x}) \stackrel{\text { def }}{=} \underset{\{\mathbf{y} \mid C(\mathbf{y}), \mathbf{y}=\alpha \mathbf{x}+(1-\alpha) \mathbf{p}\}}{\arg \min }\|\mathbf{y}-\mathbf{p}\|
$$

where $\mathbf{p}$ is an anchor point (and is set to $\mathbf{x}^{*}$ in the actual invocations), and $\|\cdot\|$ is the $l_{2}$ norm.

In the above definition, $\mathbf{y}$ lies on the line between $\mathbf{x}$ (i.e., the point before the projection) and the anchor point $\mathbf{p}$. The final projected point is the one that satisfies the constraint and also achieves the minimum $\ell_{2}$ distance from $\mathrm{p}$. To implement this projection, we perform a galloping search [9].

3.4.2 The Utility Function. We can use any $\mu$ function as long as it satisfies the condition that $\forall \mathbf{x}$ such that $F\left(\mathbf{x}^{\text {adv }}\right) \cap F\left(\mathbf{x}^{*}\right)=\emptyset$, we have $\mu(\mathbf{x})=0$. This ensures that $\mu(\mathbf{x})=0$ implies that $\mathbf{x}$ is an adversarial image. We consider two instantiations of the $\mu$ function:

- Constraint satisfaction function, i.e., $\mu(x) \stackrel{\text { def }}{=} \mathbf{1} \llbracket C(\mathbf{x}) \rrbracket$. This is the closest in spirit to the $S(\cdot)$ function used by the recent generation of decision-based adversarial attack algorithms (HSJA and QEBA) as it induces binary values for the samples.

- Average Precision. The precision at position $i$ is defined as $\operatorname{prec}(\mathbf{x}, i) \stackrel{\text { def }}{=} \frac{1}{i} \cdot\left|F\left(\mathbf{x}^{\mathrm{adv}}\right)[1: i] \cap F\left(\mathbf{x}^{*}\right)\right|$, where $A[i: j]$ takes the $i$-th to $j$-th elements (inclusive) of the array $A$. Then we define $A P(\mathbf{x})=\frac{1}{K} \cdot \sum_{i=1}^{K} \operatorname{prec}(\mathbf{x}, i)$. Then we use $\mu(\mathbf{x}) \stackrel{\text { def }}{=} A P(\mathbf{x})$. Compared with the previous choice, the AP function is more sensitive to slight changes in the ranking results, including rank position change of an image in the top- $K$ results.

We compare the performances of the two choices in the experiment.

\subsection{Optimizations}

3.5.1 Generating the Initial Adversarial Image. Previous decisionbased attack algorithms select the initial adversarial image $\mathbf{x}^{(0)}$ by randomly sampling an image in $\Omega_{+}[2,7,22]$. This essentially corresponds to sampling a random direction $\mathbf{u}$ from a (typically biased) distribution of unit vectors, and find $\mathbf{x}^{(0)}$ as $\min _{\lambda>0} \mu(\mathbf{z}+$ $\lambda \cdot \mathbf{u})=0$ by binary searching for the smallest $\lambda$.

In our framework, thanks to the fact that $\boldsymbol{\nabla}_{\mathbf{x}} J(\mathbf{x})$ exists anywhere in $\Omega_{-}$, we can generate $\mathbf{x}^{(0)}$ by performing gradient descent from $\mathbf{x}^{*}$ using $\boldsymbol{\nabla}_{\mathbf{x}} J$ till $\mu(\mathbf{x})=0$. Our choice of the adaptive direction based on $\boldsymbol{\nabla}_{\mathbf{X}} J$ is more reasonable than a fixed and typically biased random direction $\mathbf{u}$ used by HSJA or QEBA.

3.5.2 Reducing Variance of PNES Gradients. Although our gradient estimation enjoys several salient statistical properties, such as unbiasedness and consistency, its practical performance hinges upon its variance. We adopt a simple method to further reduce the variance of the gradient estimator, which is widely used in reducing the variance of the policy gradient estimate in reinforcement learning [44].

We notice that we can shift the sampled $\mu$ function values by any constant $\beta$, because

$$
\begin{aligned}
& \frac{1}{\sigma} \mathbf{E}_{\boldsymbol{\epsilon} \sim \mathrm{N}(0, \mathbf{I})} \llbracket(\mu(\mathbf{x}+\sigma \cdot \boldsymbol{\epsilon})-\beta) \cdot \boldsymbol{\epsilon} \rrbracket \\
= & \frac{1}{\sigma} \mathbf{E}_{\boldsymbol{\epsilon} \sim \mathrm{N}(0, \mathbf{I})} \llbracket \mu(\mathbf{x}+\sigma \cdot \boldsymbol{\epsilon}) \cdot \boldsymbol{\epsilon} \rrbracket-\frac{\beta}{\sigma} \mathbf{E}_{\boldsymbol{\epsilon} \sim \mathrm{N}(0, \mathbf{I})} \llbracket \boldsymbol{\epsilon} \rrbracket \\
= & \nabla_{\mathbf{X}} J(\mathbf{x})
\end{aligned}
$$

where the last step is because $\boldsymbol{\epsilon}$ is a random vector with $\mathbf{0}$ mean. This additional parameter $\beta$, which is also known as the baseline in reinforcement learning, is usually set as the average of the $\mu$ values of the samples, i.e., in our case, $\beta \stackrel{\text { def }}{=} \mathbf{E}_{\boldsymbol{\epsilon} \sim \mathrm{N}(0, \mathbf{I})} \llbracket \mu(\mathbf{x}+\sigma \cdot \boldsymbol{\epsilon}) \rrbracket$.

\subsection{Hyper-parameter Tuning}

There are two hyper-parameters in our PNES algorithms: the sampling length $\sigma$ (Eq. (4)) and the step size $\eta$ in Eq. (5). Inspired by the adaptive hyper-parameter settings in advanced stochastic gradient descent algorithms [20] and observations in our problem setting, we propose the following adaptive heuristics to adjust the two hyper-parameter values in each iteration $t$ (which is denoted as $\sigma^{(t)}$ and $\eta^{(t)}$, respectively).

Firstly, for generating the initial adversarial sample, to avoid $\mathbf{x}^{(t+1)}$ being only guided by local sampling information, we initialize $\sigma^{(t)}$ with a small value and keep increase it iteratively until $\mathbf{E} \llbracket \mu\left(\mathbf{x}^{(t+1)}\right) \rrbracket-\mathbf{E} \llbracket \mu\left(\mathbf{x}^{(t)}\right) \rrbracket>\Delta$. We set $\Delta$ as 0.3 in our implementation. Subsequently, we define a sample $\mathbf{s}_{j}$ as positive, if $\mu\left(\mathbf{s}_{j}\right) \geq \mathbf{E}_{\mathbf{s}_{\mathbf{i}}} \llbracket \mu\left(\mathbf{s}_{i}\right) \rrbracket$, and negative otherwise. Then we keep the ratio of the positive and negative weights of sampling points to around 1. More specifically, we decrease $\sigma^{(t)}$ when the ratio is higher than 2 and increase $\sigma^{(t)}$ when the ratio is lower than 0.5. And we set $\eta^{(t)}=\sigma^{(t)} / 10$.

Secondly, for the descent along the boundary, We set $\sigma^{(t)}=$ $\frac{1}{d}\left\|\mathbf{x}^{(t)}-\mathbf{x}^{*}\right\|$ and $\eta^{(t)}=\left\|\mathbf{x}^{(t)}-\mathbf{x}^{*}\right\| / \sqrt{t}$. 


\section{TRANSFERRED SELF-ADAPTIVE SAMPLING WITH AVERAGED CNN DROPOUT TECHNIQUE}

In this section, we propose a novel surrogate-based sampling method that can efficiently explore the transferability between the surrogate and the target model in a self-adaptive manner.

Employing gradients collected from surrogate models as the prior knowledge to improve the query efficiency has become a new trend in black-box adversarial attacks on classification models. However, most of the existing methods only call the surrogate model once to collect a gradient w.r.t a specific objective function as the prior knowledge in each update $[3,11]$, which does not utilize the transferability between the surrogate model and the target model efficiently. In other words, these works use the surrogate model blindly and expect that the target model is also vulnerable to the gradient collected on the surrogate model. Moreover, there is also a remarkable challenge of employing surrogate gradients on attacking retrieval models. For an image classification model, defining an efficient objective function for adversarial attacks is simple because the prediction is only dominated by some kinds of confidence score (e.g. logit scores) which can be observed locally. However, a retrieval list returned by the target retrieval model is determined by the similarity between the outputted feature embedding of the query image and the feature embeddings of candidate images stored in a large-scale database which are hard to be accessed. The current existing white-box attacks proposed to craft a valid adversarial sample by pushing away from the feature embedding vector of the query image itself [26] or drag it to a region belonging to a cluster of unrelated candidates on embedding space by the MSE loss [39]. The gradients w.r.t this kind of loss function are considered to be inefficient due to blindness or inapplicability.

To tackle the above challenges, we propose a novel usage of the surrogate model in our adversarial retrieval problem. We first use the surrogate model to generate diverse samples in the embedding space. We then derive the corresponding sampling direction in the image space by backpropagating the generated samples back through the surrogate model. Finally, we use these directions as our sampling vectors $\boldsymbol{\epsilon}_{i}$ (Eq. (4)) in the PNES algorithm. An example of this process is shown in Fig. 2. More specifically, let the surrogate model maps an image $\mathbf{x}$ to its embedding vector $s(\mathbf{x})$. We sample a batch of $n$ normalized random directions from the Gaussian distribution $U=\left\{u_{1}, \ldots, u_{n}\right\}, \forall u_{i} \in \mathbb{R}^{d}$, and multiply them with a fixed length $\rho$. This batch of random perturbations is added to the embedding vector $s(\mathbf{x})$ to produce random target embedding vectors $\mathrm{y}_{i}$. We take the $\ell_{2}$ loss between $\mathrm{y}$ and $\mathrm{y}_{i}$, and we take its gradient on the image $\mathbf{x}$ as the sampling direction, i.e., let

$$
\boldsymbol{\epsilon}_{i \mid s}=\frac{\partial \ell\left(s(\mathbf{x}), \mathbf{y}_{i}\right)}{\partial \mathbf{x}}
$$

As long as the surrogate model was trained on a dataset which has a similar semantic structure of the query image, and consequently, can extract primary characteristics of the query image efficiently, our method can give us diverse perturbation in the embedding space of the target model, which is more likely to change the value of the utility function $\mu\left(\mathbf{x}+\boldsymbol{\epsilon}_{i \mid s}\right)$. On the other hand, even if the surrogate model may present different characteristics to the

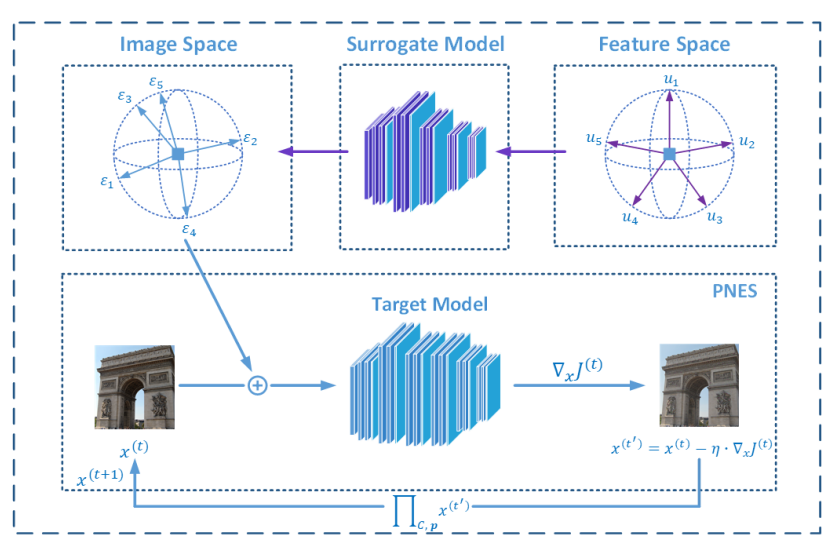

Figure 2: Overview of combining the self-adaptive sampling method with the PNES framework.

target model, the sampling direction is still likely to cause significant changes in the embedding space of the target model. Hence, we can obtain a more informative gradient estimation. These benefits will be illustrated in our empirical studies using surrogate models of different qualities.

To further increase the variability of the sampling directions generated from the surrogate model, we adapt the CNN dropout method, originally proposed to increase the transferability of adversarial examples in a white-box attack setting [49], to the introduced self-adaptive sampling method with a proposed averaging technique.

Specifically, we add random dropout layers after all the convolutional layers of the surrogate model. Due to the randomness of the dropout layer, we can deem each call of the surrogate model with dropout as calling a different surrogate model. Then for each random perturbation direction $\mathbf{u}_{i}$ in the embedding space, to refer to the gradients moving the embedding vector $s(\mathbf{x})_{j}$ in the $\mathbf{u}_{i}$ direction on different models, we obtain $m$ embeddings $s(\mathbf{x})_{j}, j \in\{1, \ldots, m\}$ by dropout and get the corresponding $\mathbf{y}_{i, j}=s(\mathbf{x})_{j}+\rho \cdot \mathbf{u}_{i}$. Then we obtain the respective $\epsilon_{i, j \mid s d}=\frac{\partial \ell\left(s(\mathrm{x})_{j}, \mathrm{y}_{i, j}\right)}{\partial \mathrm{x}}$ and average them to derive the final sampling direction:

$$
\boldsymbol{\epsilon}_{i \mid s d}=\frac{1}{m} \sum_{j=1}^{m} \frac{\partial \ell\left(s(\mathbf{x})_{j}, \mathbf{y}_{i, j}\right)}{\partial \mathbf{x}}
$$

Thanks to the random dropout and averaging technique, we can deem each $\epsilon_{i \mid s d}$ (for a fixed direction $\mathbf{u}_{i}$ in embedding space) as integrating the information of gradients from $m$ different surrogate models, which are more transferable to the target model.

\section{EXPERIMENTS}

In this section, we first detail the experimental setup. We then consider typical image retrieval models and settings (which we can control) and present the experimental results and our analyses. Next, we report the result of attacking two commercial retrieval systems to demonstrate the capability and effects of our proposed method in practice. 

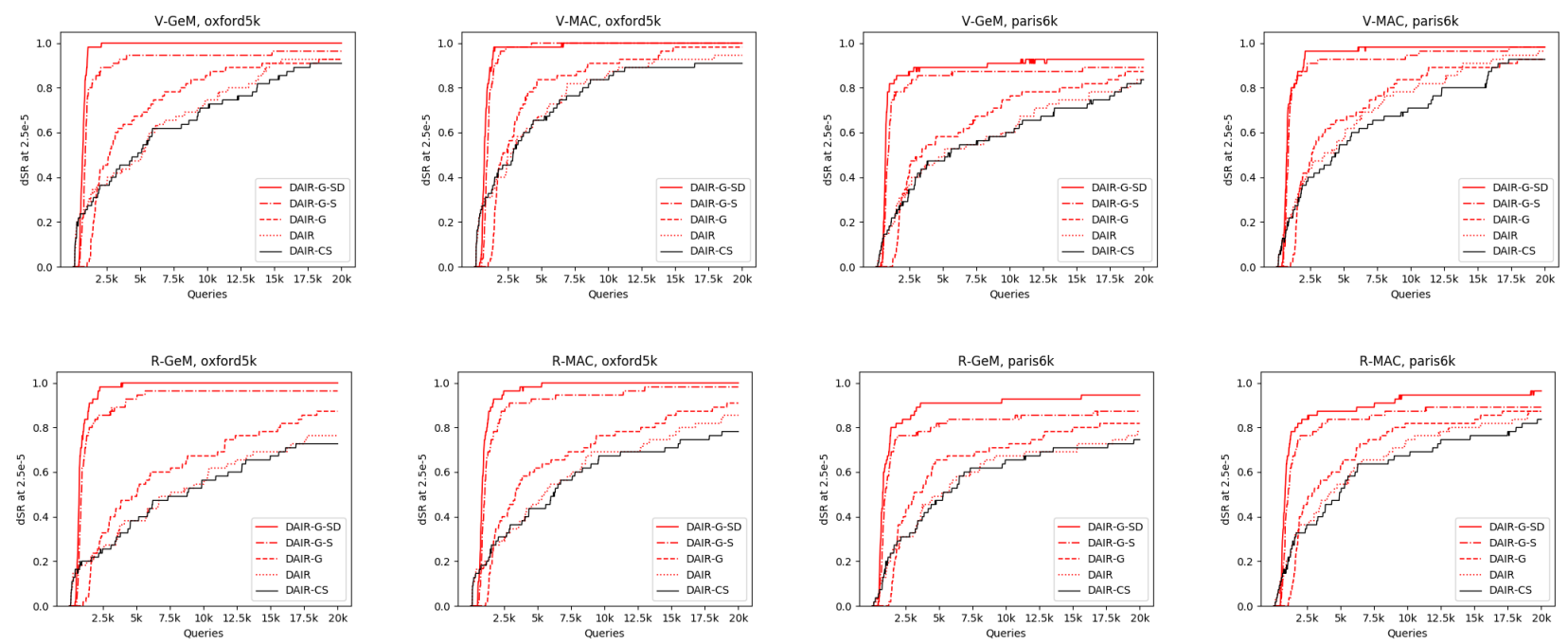

Figure 3: Plots of distortion-based success rates versus the number of queries under the distortion threshold of $2.5 \times 10^{-5} \cdot d$.

\subsection{Experimental Setup}

5.1.1 Controlled Target Retrieval Models. For the local benchmark test, we adopt the same settings as the recent works on adversarial attacks on image retrieval models [23, 26, 48]. Specifically, we considered 2 different CNN models, VGGNet16 and ResNet101, with 2 CNN-based image descriptors, GeM and MAC, to form 4 different image retrieval models. They are denoted as V-GeM, V-MAC, R-GeM, and R-MAC, respectively. All the CNN models are pretrained on the ImageNet [12] and fine-tuned on the SfM-120k of the SfM dataset [34] to learn to generate semantic-preserving deep features [33]. Then, the top- $K$ results for a query are those with the smallest $K l_{2}$ distances in the feature space. By default, we use $K=10$ for our benchmark tests.

5.1.2 Image Database and Query Images. We use the Oxford5k and Paris6k datasets [32], which are widely used in deep image retrieval research and related adversarial attacks [23, 26, 48]. Specifically, Oxford5k and Paris $6 k$ datasets both have 55 queries for 11 different landmarks in that area with 5,062 and 6,412 images as the databases, respectively. We use these queries as the original query images for our benchmark test. To demonstrate the reliability of our benchmark test, we report the mean Average Precisions (mAPs) of V-GeM, V-MAC, R-GeM, and R-MAC on the Oxford5k and Paris6k datasets in Table 1.

Table 1: Original mAPs on the Oxford5k and Paris6k.

\begin{tabular}{c||c|c}
\hline & Oxford5k & Paris6k \\
\hline V-GeM & $85.24 \%$ & $86.28 \%$ \\
\hline V-MAC & $81.45 \%$ & $88.31 \%$ \\
\hline R-GeM & $86.24 \%$ & $90.66 \%$ \\
\hline R-MAC & $81.69 \%$ & $83.55 \%$ \\
\hline
\end{tabular}

5.1.3 Attack Algorithms. We consider the following algorithm instances within our PNES framework:
DAIR-CS : This is the instance of our PNES algorithm where the constraint satisfaction function is used as the $\mu$ function, uses bi-linear sampling [22] but without any of our proposed optimization techniques. This version can be deemed as adapting the state-of-the-art decision-based attack algorithm, QEBA [22], to our problem setting.

DAIR : This is the instance of our PNES algorithm where the average precision function is used as the $\mu$ function, uses bi-linear sampling [22], but without any of our proposed optimization techniques.

DAIR-G : This is DAIR with the initial adversarial image optimization.

DAIR-G-S : This is DAIR-G with the self-adaptive sampling.

DAIR-G-SD : This is DAIR-G-S with the averaged CNN dropout.

Note that we use the variance reduction optimization for all the above methods.

We also consider the following transfer-based method:

PIRE : This is a state-of-the-art transfer attack algorithm proposed in [26].

5.1.4 Evaluation Metric. We consider three metrics: (i) the Root Mean Square Error (RMSE) measures the distortion between adversarial images $\mathbf{x}^{\text {adv }}$ and the corresponding original images $\mathbf{x}^{*}$. RMSE is the average $l_{2}$ distance between $\mathbf{x}^{\mathrm{adv}}$ and $\mathbf{x}^{*}$. (ii) Success Rate (SR) refers to the percentage of adversarial samples that successfully subvert the top- $K$ images. Note that all the statistical adversarial samples used in Table 2 have been lying on the feasible region after 2,500 queries, that is their SRs are always $100 \%$. (iii) distortionbased Success Rate (dSR) is the SR but with the additional constraint of that the norm of the perturbations is bounded by a distortion threshold. dSR measures under a small distortion threshold can better illustrate the effectiveness of multiple decision-based attack algorithms. 
Table 2: The RMSEs of attack methods on four retrieval models on Oxford5k and Paris6k datasets.

\begin{tabular}{|c|c|c|c|c|c|c|c|c|c|c|c|}
\hline \multirow{2}{*}{\multicolumn{2}{|c|}{$\frac{\text { Datasets }}{\text { Queries }}$}} & \multicolumn{5}{|c|}{ Oxford5k } & \multicolumn{5}{|c|}{ Paris6k } \\
\hline & & 2500 & 5000 & 10000 & 15000 & 20000 & 2500 & 5000 & 10000 & 15000 & 20000 \\
\hline \multirow{5}{*}{ V-GeM } & DAIR-CS & 69.55 & 56.67 & 44.46 & 37.57 & 33.05 & 86.12 & 69.79 & 54.29 & 45.82 & 40.12 \\
\hline & DAIR & 68.69 & 55.68 & 42.54 & 35.2 & 30.46 & 84.33 & 68.38 & 52.46 & 43.1 & 36.95 \\
\hline & DAIR-G & 55.92 & 46.40 & 37.61 & 32.75 & 29.27 & 69.56 & 56.42 & 45.15 & 38.91 & 34.60 \\
\hline & DAIR-G-S & 33.95 & 31.49 & 29.70 & 28.97 & 28.52 & 40.52 & 37.83 & 36.05 & 35.38 & 34.55 \\
\hline & DAIR-G-SD & 27.79 & 25.69 & 24.22 & 23.53 & 22.99 & 32.94 & 30.30 & 28.74 & 27.87 & 27.46 \\
\hline \multirow{5}{*}{ V-MAC } & DAIR-CS & 63.37 & 49.66 & 37.79 & 31.56 & 27.54 & 76.79 & 60.48 & 44.66 & 36.83 & 31.63 \\
\hline & DAIR & 61.98 & 48.63 & 36.6 & 29.81 & 25.47 & 71.23 & 54.72 & 40.47 & 32.82 & 27.87 \\
\hline & DAIR-G & 50.85 & 40.83 & 32.29 & 27.63 & 24.43 & 59.78 & 48.21 & 38.00 & 32.35 & 28.52 \\
\hline & DAIR-G-S & 28.57 & 24.87 & 22.98 & 22.27 & 21.53 & 33.69 & 29.52 & 27.15 & 26.26 & 25.68 \\
\hline & DAIR-G-SD & 26.16 & 23.01 & 21.32 & 20.39 & 19.61 & 28.68 & $25 . .04$ & 22.90 & 21.94 & 21.46 \\
\hline \multirow{5}{*}{ R-GeM } & DAIR-CS & 92.57 & 75.13 & 57.74 & 47.86 & 41.17 & 99.22 & 79.68 & 61.38 & 51.41 & 44.53 \\
\hline & DAIR & 91.66 & 72.92 & 54.71 & 44.49 & 37.67 & 97.75 & 78.84 & 59.76 & 48.87 & 41.58 \\
\hline & DAIR-G & 74.41 & 58.67 & 45.24 & 37.81 & 32.83 & 80.38 & 62.83 & 49.13 & 41.68 & 36.77 \\
\hline & DAIR-G-S & 36.85 & 33.61 & 31.47 & 30.48 & 29.86 & 41.65 & 37.58 & 34.60 & 33.17 & 32.39 \\
\hline & DAIR-G-SD & 30.78 & 27.74 & 25.81 & 24.93 & 24.39 & 33.23 & 29.56 & 27.16 & 25.97 & 25.22 \\
\hline \multirow{5}{*}{ R-MAC } & DAIR-CS & 86.63 & 68.33 & 50.86 & 41.47 & 35.06 & 94.50 & 73.59 & 54.93 & 44.92 & 38.42 \\
\hline & DAIR & 85.67 & 66.59 & 47.65 & 37.31 & 30.78 & 88.99 & 68.93 & 50.10 & 40.09 & 33.33 \\
\hline & DAIR-G & 69.21 & 53.40 & 40.41 & 33.33 & 28.60 & 73.82 & 55.69 & 42.32 & 35.05 & 30.26 \\
\hline & DAIR-G-S & 34.83 & 30.69 & 28.41 & 27.33 & 26.69 & 37.84 & 32.72 & 29.98 & 28.82 & 28.19 \\
\hline & DAIR-G-SD & 29.69 & 25.59 & 22.88 & 21.71 & 21.03 & 32.84 & 28.19 & 24.92 & 23.31 & 22.39 \\
\hline
\end{tabular}

5.1.5 Implementation Details. For the bilinear sampling [22], we use the sub-sample rate of 0.5 . The number of samples used for gradient estimation, $B$, is set to 20 . We set the maximum number of queries to 20,000 for the benchmark test.

For the surrogate-based sampling, we use the convolutional layers of an AlexNet trained only on the ImageNet as the backbone of the surrogate model subsequent with an AdaptiveAveragePool layer to unify the dimension of feature vectors w.r.t input query images with different sizes. Moreover, to simplify the tuning process of the lengths of Gaussian noises adding on the feature vectors output by the surrogate model, we add a feature normalization layer after the AdaptiveAveragePool layer to keep the $l_{2}$ norms as 1 . We set the lengths of Gaussian noises adding on the feature vectors as 1 . We abbreviate this model structure as A-Adap in the following context. For DAIR-G-SD, we set the dropout rate as 0.4 and $m=10$ of the batch size for the averaged dropout by default.

For the transfer-based attack PIRE, we use the identical implementation details reported in [26]. Specifically, we use Adam as the optimizer, with a learning rate $\alpha=10^{-4}$ to optimize the perturbation on the surrogate model for 500 iterations. Then, we multiply the final perturbation by 7 for the subsequent transfer-based attack. Although the authors propose to use 10 as the enlarged factor by default, we consider the perturbation will become too remarkable and lost the significance of testing.

\subsection{Comparison Among DAIR Variants}

In Table 2, we report the RMSEs of adversarial images generated by all the DAIR methods on the Oxford5k and Paris6k datasets. The results in the table can also be deemed as performing ablation studies of various optimizations we proposed.

We can see that (i) DAIR-G-S and DAIR-G-SD significantly outperform all other methods in all settings. Moreover, the RMSEs collected by DAIR-G-SD at 2,500 queries are even smaller than DAIR-CS collected at 20,000 queries in all settings. This observation demonstrates that our proposed surrogate-based framework can remarkably improve the query efficiency of the attack. (ii) using the AP function as the $\mu$ function gives superior performance than using the constraint satisfaction function (as used by DAIR-CS) in all settings. (iii) generating an initial adversarial sample for DAIR in DAIR-G can further reduce the used queries by 2,500 to achieve a comparable RMSE on average. (iv) introducing the proposed averaged CNN dropout method can further improve the performances of DAIR-G-S.

In addition, we show the plots of the distortion-based success rates (dSRs) versus the number of queries with a distortion threshold of $2.5 \times 10^{-5} \cdot d$ for all the reported settings in Fig. 3 . We can see that DAIR-G-SD outperforms all other methods and can achieve a high success rate even with a small number of queries.

Table 3: Success rates of PIRE on Paris6k.

\begin{tabular}{c||r|r|r|r}
\hline Surrogate $\backslash$ Target & V-GeM & V-MAC & R-GeM & R-MAC \\
\hline V-GeM & - & $100.00 \%$ & $78.18 \%$ & $74.54 \%$ \\
\hline V-MAC & $100.00 \%$ & - & $69.09 \%$ & $63.64 \%$ \\
\hline R-GeM & $16.36 \%$ & $20.00 \%$ & - & $100.00 \%$ \\
\hline R-MAC & $14.45 \%$ & $16.36 \%$ & $80.00 \%$ & - \\
\hline \hline A-Adap & $63.63 \%$ & $67.27 \%$ & $36.36 \%$ & $36.36 \%$ \\
\hline
\end{tabular}

Table 4: RMSEs of PIRE on Paris6k.

\begin{tabular}{l||c|c|c|c||c}
\hline Target & V-GeM & V-MAC & R-GeM & R-MAC & A-Adap \\
\hline RMSE & 80.46 & 78.60 & 77.01 & 70.27 & 93.99 \\
\hline
\end{tabular}




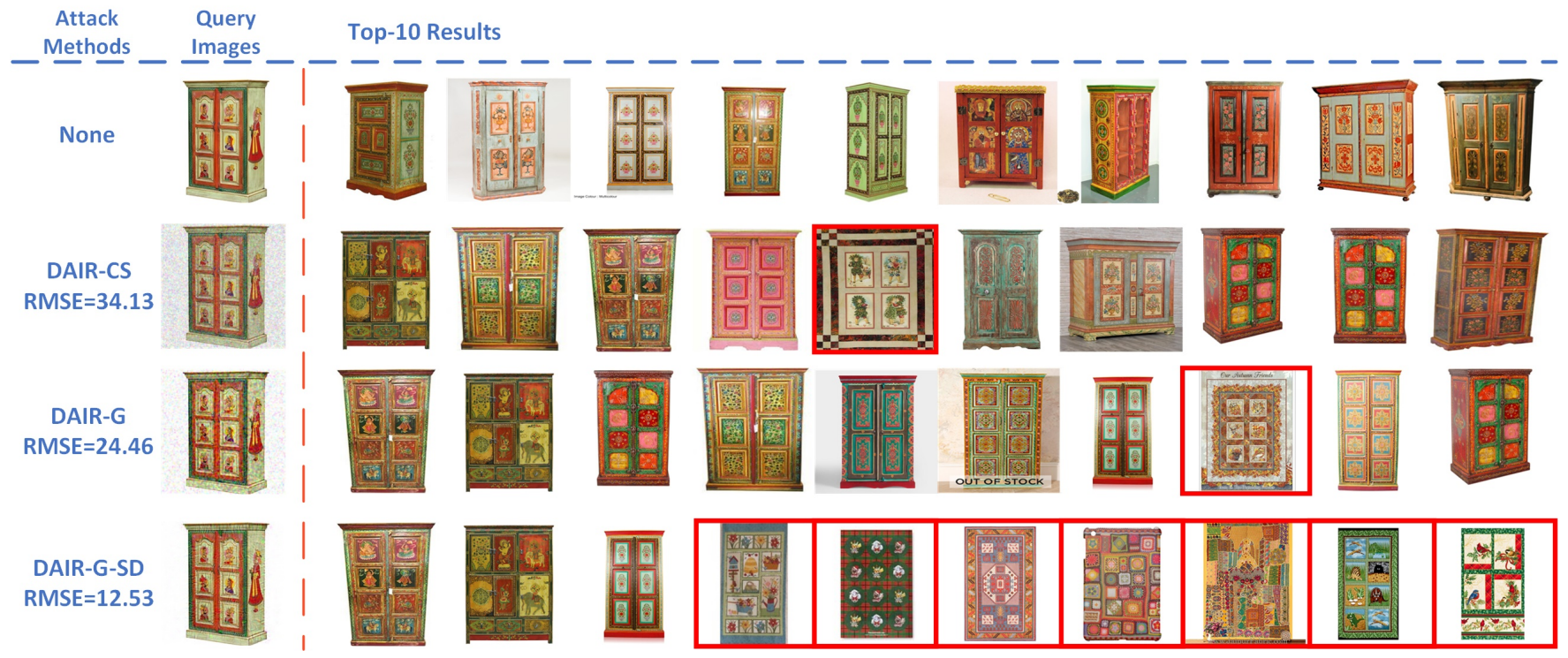

Figure 4: An example of attacking the Bing Visual Search API. Note that images in red frames indicate that they are different type of product (i.e., carpet) to the original query image (i.e., wardrobe).

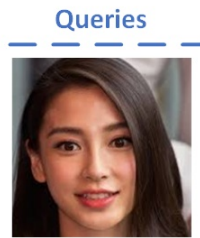

Original Image

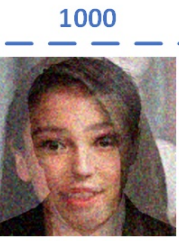

RMSE $=51.02$

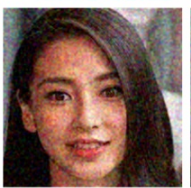

RMSE $=35.01$

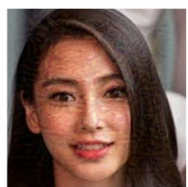

RMSE $=6.94$

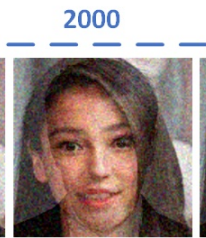

RMSE $=40.50$

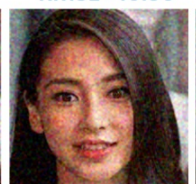

RMSE $=18.21$

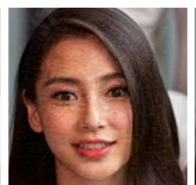

RMSE $=4.80$
3000

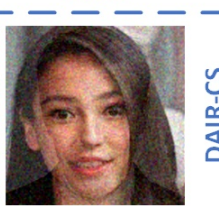

RMSE $=33.99$

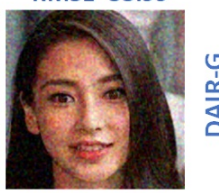

RMSE $=18.18$

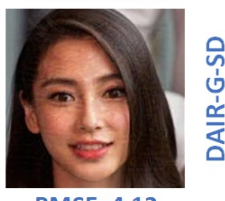

RMSE=4.12

Figure 5: An example of attacking the Face++ Face Search API under different number of queries.

\subsection{Comparing with Transfer-based Attacks}

To understand the relative effectiveness of our proposed attack methods, we compare with the state-of-the-art transfer-based algorithm PIRE. We first consider the ideal situation where the attacker has access to all of the training and database images. And the attacker trains a surrogate model and computes its corresponding adversarial image using PIRE, and then evaluates its transfer success rate on another target model. The result of success rates among different models is shown in Table 3 (specifically the first part of

the table). We also report the RMSEs of perturbations generated on the surrogates in Table 4.

Next, we consider a more realistic setting where we employ our defined A-Adap model (see "Implementation Details") as the surrogate to attack the four benchmark target models by PIRE (See the "A-Adap" row/column in Table 3 and 4). Both the success rate and the perturbation required are worse compared with the ideal setting. This shows that it is hard to generate an imperceptible adversarial perturbation which is also reliable for the attack by PIRE without knowing the detailed information of the target model.

\subsection{Effect of the Averaged CNN Dropout}

To demonstrate that our proposed averaged CNN dropout can further improve the capability of the $\mathrm{CNN}$ dropout method proposed by [49] in the self-adaptive sampling method, we study the influence of the batch size $m$ for the averaged CNN dropout. In Table 5, we report the RMSEs and used queries of generating valid initial adversarial perturbations by DAIR-G-SD with $m=1,5,10$, and 20 for V-GeM on the Oxford5k dataset. It should be noticed that averaged $\mathrm{CNN}$ dropout can be regarded as the original CNN dropout method when $m=1$

Table 5: Influence of batch size on averaged CNN dropout.

\begin{tabular}{c|c|c|c|c}
\hline Batch Size & $\mathrm{m}=1$ & $\mathrm{~m}=5$ & $\mathrm{~m}=10$ & $\mathrm{~m}=20$ \\
\hline RMSE & 41.13 & 38.83 & 37.65 & 37.63 \\
\hline Queries & 1060.80 & 796.47 & 688.43 & 661.12 \\
\hline
\end{tabular}

The result illustrates that increasing the batch size for the CNN dropout can reduce the RMSEs and the queries of generating initial adversarial perturbations simultaneously. To tradeoff between the time complexity and the performance, we set $m=10$ for DAIR-GSD by default. 
Table 6: The RMSEs and dSRs of attack methods on attacking Bing Visual Search and Face++ Face Search APIs.

\begin{tabular}{|c|c|c|c|c|c|c|c|c|c|c|c|c|c|c|c|c|}
\hline Type & \multicolumn{4}{|c|}{ Bing: Building } & \multicolumn{4}{|c|}{ Bing: Daily Supplies } & \multicolumn{4}{|c|}{ Bing: Portrait } & \multicolumn{4}{|c|}{ Face++: Face } \\
\hline Queries & \multicolumn{2}{|c|}{500} & \multicolumn{2}{|c|}{3000} & \multicolumn{2}{|c|}{500} & \multicolumn{2}{|c|}{3000} & \multicolumn{2}{|c|}{500} & \multicolumn{2}{|c|}{3000} & \multicolumn{2}{|c|}{500} & \multicolumn{2}{|c|}{3000} \\
\hline Metrics & RMSE & dSR & RMSE & dSR & RMSE & dSR & RMSE & dSR & RMSE & dSR & RMSE & dSR & RMSE & $\mathrm{dSR}$ & RMSE & dSR \\
\hline DAIR-CS & 88.60 & 0 & 69.97 & 0 & 68.40 & 0 & 50.78 & 0.4 & 84.01 & 0 & 66.64 & 0.1 & 39.04 & 0 & 26.40 & 0.3 \\
\hline DAIR-G & 32.64 & 0.1 & 35.34 & 0.9 & 23.14 & 0.4 & 30.91 & 1 & 32.36 & 0.1 & 33.44 & 0.8 & 17.32 & 0.3 & 16.74 & 0.3 \\
\hline DAIR-G-SD & 20.94 & 0.8 & 17.95 & 1 & 12.92 & 1 & 11.92 & 1 & 17.03 & 0.7 & 18.99 & 1 & 8.81 & 0.8 & 6.64 & 0.8 \\
\hline
\end{tabular}

\subsection{Attacking Commercial Systems}

To demonstrate the effectiveness and practicality of our proposed attack methods, we applied DAIR-CS, DAIR-G, and DAIR-G-SD methods to attack the Bing Visual Search API ${ }^{4}$ and Face++ Face Search API ${ }^{5}$. We set $K=50$ for Bing and $K=5$ for Face ++ , as Face ++ only returns at most 5 images as the search results.

For the test on Bing, we prepare three types of images including building, portrait, and daily supplies as target queries. Each type of image is constituted by 10 different images collected from real life. Although we do not know the training data or the internal structure of the online systems, we propose to use a CNN-based model, which only trained on a dataset with a similar semantic structure of the query image, as the surrogate for attacking each type of the query image by DAIR-G-SD. Specifically, we use our implemented AAdap model trained on the ImageNet as the surrogate for attacking all the daily supplies images. And we use the same A-Adap model trained on the ImageNet but also fine-tuned on the SfM dataset [34] as the surrogate for the building query images. We also introduce Inception ResNet [35] trained on the VGGFace2 dataset [4] for the portrait images. All these three models are preprocessed by our aforementioned procedure, that is we only use the CNN part of the models subsequent with an AdaptiveAveragePool layer and a feature normalization layer. The dropout rates we used for these three models are $0.2,0.2$, and 0.05 , respectively. And the averaged dropout batch sizes are all set to 10 by default.

Face++ provides its well-trained face retrieval model to users and requires users to set up their own face candidate datasets for retrieval. In our implementation, we select 5,000 face images from the MS-Celeb dataset [45] which includes 5.05 million face images from 79,077 different identities, to build our test dataset on Face++. Specifically, we select 250 different identities and choose 20 related face images for each identity. We also collect 10 different face images as queries. We use the same Inception ResNet surrogate model used by attacking portrait images on Bing API for attacking Face++ by DAIR-G-SD. It should be noticed that Face++ will first detect whether the query image is a valid face image, which means that it is hard to define an initial adversarial sample by the random search method in DAIR-CS. Therefore, we select a source image of another identity with a similar structure as the query image as the initial adversarial sample for the subsequent boundary attack. However, both DAIR-G and DAIR-G-SD can successfully generate a valid initial adversarial sample from the target query image, which is easier to be implemented in practice.

In Table 6, we report the RMSEs and dSRs of adversarial perturbations generated by the three compared methods for the four

\footnotetext{
${ }^{4}$ https://www.microsoft.com/en-us/bing/apis/bing-visual-search-api

${ }^{5}$ https://www.faceplusplus.com.cn/face-searching/
}

types of images at 500 and 3,000 queries. Here, we set the distortion threshold as $1 \times 10^{-4} \cdot d$. We can see that our methods can successfully attack these two arguably different systems. Specifically, the DAIR-G-SD algorithm achieves high success rates and the lowest RMSEs under all the settings.

Case Studies We give two case studies for attacking the two online systems to visually represent the practicalities of our proposed methods. In Fig. 4, we show an example of attacking the retrieval result of a daily supplies image returned by Bing. Each of the adversarial samples is collected at 3,000 queries. We can see that all of the three adversarial samples can successfully subvert the retrieval result of the original query image, which reveals the vulnerability of Bing against the decision-based attacks. Moreover, we can also see that the adversarial sample crafted by DAIR-G-SD is much more imperceptible than the adversarial sample generated by the baseline method, which demonstrates the superior performance of our proposed method. In Fig. 5, we present a qualitative example process of attacking the Face++ by the three compared methods. Due to the requirement of a source face image for DAIR-CS, the generated adversarial samples are remarkable to human perceptions. As a comparison, the adversarial samples generated by our proposed method are reasonably imperceptible at a very early stage. This example also demonstrates the importance of generating a valid adversarial sample from the target query image rather than randomly selecting a sample as the initial point for the subsequent boundary attack.

\section{CONCLUSIONS}

In this paper, we proposed query-efficient decision-based attack methods to image retrieval systems. Our attack methods require merely the ability to issue queries to the system and obtain the identities of the top- $K$ retrieved images. Our method is based on optimizing a smoothed utility function using Projected Natural Evolution Strategies. Moreover, we proposed an efficient surrogatebased sampling strategy with an averaged CNN dropout technique that can improve the query efficiency drastically. Extensive experimental evaluation, including both image retrieval benchmarks and two commercial systems, showcases the effectiveness and the practicability of our proposed methods.

Acknowledgements The work was supported in part by ARC Discovery Projects 180103411 and 220101762, Natural Science Foundation of China (grant no.61876038), Dongguan Social Science and Technology Development Key Project (grant no. 2020507140146), Dongguan University of Technology under project (grant no. KCYKYQD2017003), Guangdong Basic and Applied Basic Research Foundation (grant no. 2020B1515120028), and Guangdong Peral River Recruitment Program of Talents (grant no. 2019ZT08X603). 


\section{REFERENCES}

[1] Artem Babenko and Victor S. Lempitsky. 2015. Aggregating Local Deep Features for Image Retrieval. In ICCV. IEEE Computer Society, 1269-1277.

[2] Wieland Brendel, Jonas Rauber, and Matthias Bethge. 2018. Decision-Based Adversarial Attacks: Reliable Attacks Against Black-Box Machine Learning Models. In ICLR (Poster). OpenReview.net.

[3] Thomas Brunner, Frederik Diehl, Michael Truong-Le, and Alois C. Knoll. 2019 Guessing Smart: Biased Sampling for Efficient Black-Box Adversarial Attacks. In ICCV. IEEE, 4957-4965.

[4] Qiong Cao, Li Shen, Weidi Xie, Omkar M. Parkhi, and Andrew Zisserman. 2018 VGGFace2: A Dataset for Recognising Faces across Pose and Age. In FG. IEEE Computer Society, 67-74.

[5] Nicholas Carlini and David A. Wagner. 2017. Towards Evaluating the Robustness of Neural Networks. In IEEE Symposium on Security and Privacy. IEEE Computer Society, 39-57.

[6] Chenyi Chen, Ari Seff, Alain L. Kornhauser, and Jianxiong Xiao. 2015. DeepDriving: Learning Affordance for Direct Perception in Autonomous Driving. In ICCV. IEEE Computer Society, 2722-2730.

[7] Jianbo Chen, Michael I. Jordan, and Martin J. Wainwright. 2020. HopSkipJumpAttack: A Query-Efficient Decision-Based Attack. In IEEE Symposium on Security and Privacy. IEEE, 1277-1294.

[8] Pin-Yu Chen, Huan Zhang, Yash Sharma, Jinfeng Yi, and Cho-Jui Hsieh. 2017 ZOO: Zeroth Order Optimization Based Black-box Attacks to Deep Neural Networks without Training Substitute Models. In AISec@CCS. ACM, 15-26.

[9] Minhao Cheng, Thong Le, Pin-Yu Chen, Huan Zhang, Jinfeng Yi, and Cho-Jui Hsieh. 2019. Query-Efficient Hard-label Black-box Attack: An Optimization-based Approach. In ICLR (Poster). OpenReview.net.

[10] Minhao Cheng, Simranjit Singh, Patrick H. Chen, Pin-Yu Chen, Sijia Liu, and Cho-Jui Hsieh. 2020. Sign-OPT: A Query-Efficient Hard-label Adversarial Attack. In ICLR. OpenReview.net.

[11] Shuyu Cheng, Yinpeng Dong, Tianyu Pang, Hang Su, and Jun Zhu. 2019. Improving Black-box Adversarial Attacks with a Transfer-based Prior. In NeurIPS 10932-10942.

[12] Jia Deng, Wei Dong, Richard Socher, Li-Jia Li, Kai Li, and Fei-Fei Li. 2009. ImageNet: A large-scale hierarchical image database. In CVPR. IEEE Computer Society, 248-255.

[13] Yinpeng Dong, Hang Su, Baoyuan Wu, Zhifeng Li, Wei Liu, Tong Zhang, and Jun Zhu. 2019. Efficient Decision-Based Black-Box Adversarial Attacks on Face Recognition. In CVPR. Computer Vision Foundation / IEEE, 7714-7722.

[14] Ian J. Goodfellow, Jonathon Shlens, and Christian Szegedy. 2015. Explaining and Harnessing Adversarial Examples. In ICLR (Poster).

[15] Jiatao Gu, James Bradbury, Caiming Xiong, Victor O. K. Li, and Richard Socher 2018. Non-Autoregressive Neural Machine Translation. In ICLR (Poster). OpenReview.net.

[16] Kaiming He, Xiangyu Zhang, Shaoqing Ren, and Jian Sun. 2016. Deep Residual Learning for Image Recognition. In CVPR. IEEE Computer Society, 770-778.

[17] Andrew Ilyas, Logan Engstrom, Anish Athalye, and Jessy Lin. 2018. Black-box Adversarial Attacks with Limited Queries and Information. In ICML (Proceedings of Machine Learning Research, Vol. 80). PMLR, 2142-2151.

[18] Yannis Kalantidis, Clayton Mellina, and Simon Osindero. 2016. CrossDimensional Weighting for Aggregated Deep Convolutional Features. In ECCV Workshops (1) (Lecture Notes in Computer Science, Vol. 9913). Springer, 685-701.

[19] Andrej Karpathy, George Toderici, Sanketh Shetty, Thomas Leung, Rahul Suk thankar, and Fei-Fei Li. 2014. Large-Scale Video Classification with Convolutional Neural Networks. In CVPR. IEEE Computer Society, 1725-1732.

[20] Diederik P. Kingma and Jimmy Ba. 2015. Adam: A Method for Stochastic Optimization. In 3rd International Conference on Learning Representations, ICLR 2015, San Diego, CA, USA, May 7-9, 2015, Conference Track Proceedings. http://arxiv.org/abs/1412.6980

[21] Alexey Kurakin, Ian J. Goodfellow, and Samy Bengio. 2017. Adversarial examples in the physical world. In ICLR (Workshop). OpenReview.net.

[22] Huichen Li, Xiaojun Xu, Xiaolu Zhang, Shuang Yang, and Bo Li. 2020. QEBA Query-Efficient Boundary-Based Blackbox Attack. In CVPR. IEEE, 1218-1227.

[23] Jie Li, Rongrong Ji, Hong Liu, Xiaopeng Hong, Yue Gao, and Qi Tian. 2019. Universal Perturbation Attack Against Image Retrieval. In ICCV. IEEE, 48984907

[24] Sijia Liu, Bhavya Kailkhura, Pin-Yu Chen, Pai-Shun Ting, Shiyu Chang, and Lisa Amini. 2018. Zeroth-Order Stochastic Variance Reduction for Nonconvex Optimization. In NeurIPS. 3731-3741.

[25] Yanpei Liu, Xinyun Chen, Chang Liu, and Dawn Song. 2017. Delving into Transferable Adversarial Examples and Black-box Attacks. In ICLR (Poster). OpenReview.net.

[26] Zhuoran Liu, Zhengyu Zhao, and Martha A. Larson. 2019. Who's Afraid of Adversarial Queries?: The Impact of Image Modifications on Content-based Image Retrieval. In Proceedings of the 2019 on International Conference on Multimedia Retrieval, ICMR 2019, Ottawa, ON, Canada, June 10-13, 2019. 306-314. https: //doi.org/10.1145/3323873.3325052
[27] Jonathan Long, Evan Shelhamer, and Trevor Darrell. 2015. Fully convolutional networks for semantic segmentation. In CVPR. IEEE Computer Society, 34313440 .

[28] Xinzhu Ma, Zhihui Wang, Haojie Li, Pengbo Zhang, Wanli Ouyang, and Xin Fan. 2019. Accurate Monocular 3D Object Detection via Color-Embedded 3D Reconstruction for Autonomous Driving. In ICCV. IEEE, 6850-6859.

[29] Aleksander Madry, Aleksandar Makelov, Ludwig Schmidt, Dimitris Tsipras, and Adrian Vladu. 2018. Towards Deep Learning Models Resistant to Adversarial Attacks. In ICLR (Poster). OpenReview.net.

[30] Seyed-Mohsen Moosavi-Dezfooli, Alhussein Fawzi, Omar Fawzi, and Pascal Frossard. 2017. Universal Adversarial Perturbations. In CVPR. IEEE Computer Society, 86-94.

[31] Seyed-Mohsen Moosavi-Dezfooli, Alhussein Fawzi, and Pascal Frossard. 2016. DeepFool: A Simple and Accurate Method to Fool Deep Neural Networks. In CVPR. IEEE Computer Society, 2574-2582.

[32] Filip Radenovic, Ahmet Iscen, Giorgos Tolias, Yannis Avrithis, and Ondrej Chum. 2018. Revisiting Oxford and Paris: Large-Scale Image Retrieval Benchmarking. In CVPR. IEEE Computer Society, 5706-5715.

[33] Filip Radenovic, Giorgos Tolias, and Ondrej Chum. 2019. Fine-Tuning CNN Image Retrieval with No Human Annotation. IEEE Trans. Pattern Anal. Mach. Intell. 41, 7 (2019), 1655-1668.

[34] Johannes L. Schönberger, Filip Radenovic, Ondrej Chum, and Jan-Michael Frahm. 2015. From single image query to detailed 3D reconstruction. In CVPR. IEEE Computer Society, 5126-5134.

[35] Florian Schroff, Dmitry Kalenichenko, and James Philbin. 2015. FaceNet: A unified embedding for face recognition and clustering. In CVPR. IEEE Computer Society, $815-823$

[36] Karen Simonyan and Andrew Zisserman. 2015. Very Deep Convolutional Networks for Large-Scale Image Recognition. In ICLR

[37] Christian Szegedy, Wei Liu, Yangqing Jia, Pierre Sermanet, Scott E. Reed, Dragomir Anguelov, Dumitru Erhan, Vincent Vanhoucke, and Andrew Rabinovich. 2015. Going deeper with convolutions. In CVPR. IEEE Computer Society, $1-9$

[38] Christian Szegedy, Wojciech Zaremba, Ilya Sutskever, Joan Bruna, Dumitru Erhan, Ian J. Goodfellow, and Rob Fergus. 2014. Intriguing properties of neural networks. In ICLR (Poster).

[39] Giorgos Tolias, Filip Radenovic, and Ondrej Chum. 2019. Targeted Mismatch Adversarial Attack: Query With a Flower to Retrieve the Tower. In ICCV. IEEE, 5036-5045.

[40] Giorgos Tolias, Ronan Sicre, and Hervé Jégou. 2016. Particular object retrieval with integral max-pooling of CNN activations. In ICLR (Poster).

[41] Ashish Vaswani, Noam Shazeer, Niki Parmar, Jakob Uszkoreit, Llion Jones, Aidan N. Gomez, Lukasz Kaiser, and Illia Polosukhin. 2017. Attention is All you Need. In NIPS. 5998-6008.

[42] Ji Wan, Dayong Wang, Steven Chu-Hong Hoi, Pengcheng Wu, Jianke Zhu, Yongdong Zhang, and Jintao Li. 2014. Deep Learning for Content-Based Image Retrieval: A Comprehensive Study. In ACM Multimedia. ACM, 157-166.

[43] Daan Wierstra, Tom Schaul, Tobias Glasmachers, Yi Sun, Jan Peters, and Jürgen Schmidhuber. 2014. Natural evolution strategies. f. Mach. Learn. Res. 15, 1 (2014), 949-980. http://dl.acm.org/citation.cfm?id=2638566

[44] Ronald J. Williams. 1992. Simple Statistical Gradient-Following Algorithms for Connectionist Reinforcement Learning. Mach. Learn. 8 (1992), 229-256. https://doi.org/10.1007/BF00992696

[45] Xiang Wu, Ran He, Zhenan Sun, and Tieniu Tan. 2018. A Light CNN for Deep Face Representation With Noisy Labels. IEEE Trans. Inf. Forensics Secur. 13, 11 (2018), 2884-2896.

[46] Rongkai Xia, Yan Pan, Hanjiang Lai, Cong Liu, and Shuicheng Yan. 2014. Supervised Hashing for Image Retrieval via Image Representation Learning. In $A A A I$. AAAI Press, 2156-2162.

[47] Erkun Yang, Tongliang Liu, Cheng Deng, and Dacheng Tao. 2020. Adversarial Examples for Hamming Space Search. IEEE Trans. Cybern. 50, 4 (2020), 1473-1484.

[48] Guoping Zhao, Mingyu Zhang, Jiajun Liu, and Ji-Rong Wen. 2019. Unsupervised Adversarial Attacks on Deep Feature-based Retrieval with GAN. CoRR abs/1907.05793 (2019).

[49] Yaoyao Zhong and Weihong Deng. 2021. Towards Transferable Adversarial Attack Against Deep Face Recognition. IEEE Trans. Inf. Forensics Secur. 16 (2021), $1452-1466$.

[50] Mo Zhou, Zhenxing Niu, Le Wang, Qilin Zhang, and Gang Hua. 2020. Adversarial Ranking Attack and Defense. CoRR abs/2002.11293 (2020). 